\title{
Can we improve cognitive function among adults with osteoarthritis by increasing moderate-to-vigorous physical activity and reducing sedentary behaviour? Secondary analysis of the MONITOR-OA study
}

Ryan S. Falck', John R. Best ${ }^{1}$, Linda C. Li' ${ }^{2}$ Patrick C. Y. Chan ${ }^{1}$, Lynne M. Feehan² and Teresa Liu-Ambrose ${ }^{1 *}$

\begin{abstract}
Background: Preliminary evidence suggests osteoarthritis is a risk factor for cognitive decline. One potential reason is $87 \%$ of adults with osteoarthritis are inactive, and low moderate-to-vigorous physical activity and high sedentary behaviour are each risk factors for cognitive decline. Thus, we investigated whether a community-based intervention to increase moderate-to-vigorous physical activity and reduce sedentary behaviour could improve cognitive function among adults with osteoarthritis.

Methods: This was a secondary analysis of a six month, proof-of-concept randomized controlled trial of a communitybased, technology-enabled counselling program to increase moderate-to-vigorous physical activity and reduce sedentary behaviour among adults with knee osteoarthritis. The Immediate Intervention $(n=30)$ received a Fitbit ${ }^{\oplus}$ Flex $^{\mathrm{TM}}$ and four bi-weekly activity counselling sessions; the Delayed Intervention $(n=31)$ received the same intervention two months later. We assessed episodic memory and working memory using the National Institutes of Health Toolbox Cognition Battery. Between-group differences (Immediate Intervention vs. Delayed Intervention) in cognitive performance were evaluated following the primary intervention (i.e., Baseline - 2 Months) using intention-to-treat.

Results: The intervention did not significantly improve cognitive function; however, we estimated small average improvements in episodic memory for the Immediate Intervention vs. Delayed Intervention (estimated mean difference: $1.27 ; 95 \% \mathrm{Cl}[-9.27,11.81] ; d=0.10)$.

Conclusion: This small study did not show that a short activity promotion intervention improved cognitive health among adults with osteoarthritis. However, the effects of increased moderate-to-vigorous physical activity and reduced sedentary behaviour are likely to be small and thus we recommend subsequent studies use larger sample sizes and measure changes in cognitive function over longer intervals.
\end{abstract}

Trial registration number: ClinicalTrials.gov Protocol Registration System: NCT02315664; registered 12 December, 2014; https:/clinicaltrials.gov/ct2/show/NCT02315664?cond=NCT02315664\&rank=1

Keywords: Physical activity, Sedentary behaviour, Cognitive function, Osteoarthritis

\footnotetext{
* Correspondence: teresa.ambrose@ubc.ca

${ }^{1}$ Faculty of Medicine, Aging, Mobility and Cognitive Neuroscience Laboratory,

Djavad Mowafaghian Centre for Brain Health, Department of Physical

Therapy, University of British Columbia, 212-2177 Wesbrook Mall, Vancouver,

BC V6T 1Z3, Canada

Full list of author information is available at the end of the article
}

(c) The Author(s). 2018 Open Access This article is distributed under the terms of the Creative Commons Attribution 4.0 International License (http://creativecommons.org/licenses/by/4.0/), which permits unrestricted use, distribution, and reproduction in any medium, provided you give appropriate credit to the original author(s) and the source, provide a link to the Creative Commons license, and indicate if changes were made. The Creative Commons Public Domain Dedication waiver (http://creativecommons.org/publicdomain/zero/1.0/) applies to the data made available in this article, unless otherwise stated. 


\section{Background}

One new diagnosis of osteoarthritis (OA) occurs every 60 s, such that $9.6 \%$ of all men and $18.0 \%$ of all women over age 60 have symptomatic OA $[1,2]$. Of those living with OA, $80 \%$ will have limitations in movement and $25 \%$ cannot perform their major daily activities of life [2]. The pain of OA is associated with 1) reduced physical function and mobility [3]; and 2) increased frailty and falls risk [4]. While total knee replacement is effective for end-stage OA [5], it does not uniformly restore joint function, and $20 \%$ of patients continue to experience pain [6].

Preliminary evidence also suggests $\mathrm{OA}$ is associated with an increased risk of cognitive decline and dementia [7]. Although the association between OA and dementia is still under investigation [8], animal models suggest that peripheral inflammation associated with OA may trigger neural inflammation and induce Alzheimer's disease pathology-the most common form of dementia [9]. Given that the number of cases of OA and dementia are each increasing as the population of older adults continues to grow $[10,11]$, there is an urgent need for effective treatment strategies for OA symptoms since this may also help reduce dementia prevalence.

Two frontline strategies for improving OA symptoms are increasing moderate-to-vigorous physical activity and reducing sedentary behaviour [12-16]. Briefly, moderate-to-vigorous physical activity (MVPA) refers to any behaviour which incurs $\geq 3.0$ metabolic equivalents (METs), while sedentary behaviour (SB) refers to any behaviour which incurs $\leq 1.5 \mathrm{METs}$ and occurs while sitting or lying down. While OA is linked to declines in joint protective biomarkers such as lubricin and pituitary adenylate cyclase-activating polypeptide (PACAP), and increases in inflammatory markers and apoptotic signaling [17, 18], animal models of OA indicate that MVPA can 1) promote lubricin synthesis [19-21]; 2) down-regulate apoptotic signalling [19]; 3) down-regulate inflammatory markers of OA including interleukin-1 [21]; and 4) may stimulate the production of PACAP [22]. Increased MVPA can also improve strength and balance in adults with arthritis [23], reduce the risk of falls [24], and reduce OA symptoms such as pain, fatigue, and joint stiffness [25]. While less is known about how SB may impact the symptoms of OA, epidemiological evidence suggests that reduced SB is associated with better physical function in adults with $\mathrm{OA}-$ independent of MVPA time [12, 13].

There is also strong evidence that both high MVPA and low SB are neuroprotective [26, 27]. Animal models suggest that MVPA reduces pro-inflammatory markers and amyloid $\beta$ protein levels in transgenic mice predisposed to Alzheimer's disease [28], and human epidemiological data consistently indicates that MVPA is associated with better cognitive function and a lower risk of cognitive decline [27]. Greater amounts of SB may negatively impact the cellular mechanisms by which MVPA improves cognitive health [29], and may alter the connectivity of the brain such that cognitive function worsens with greater SB [30]. As such, current guidelines suggest that all adults should engage in $\geq 150 \mathrm{~min}$ of MVPA each week and limit discretionary SB as much as possible [26].

Given that 1) increasing MVPA and reducing SB promotes cellular mechanisms which reduce OA associated inflammatory and apoptotic responses [19-22, 28, 29]; and 2) OA associated inflammation and apoptotic signalling increases dementia risk [9], it is plausible that increasing MVPA and reducing SB is an effective frontline dementia prevention strategy for adults with OA. Unfortunately, the uptake of knowledge about the importance of MVPA and SB for controlling OA symptoms and reducing dementia risk has been slow. Among adults living with OA, $87 \%$ do not meet current recommendations of $\geq 150 \mathrm{~min} /$ week of MVPA [31], and people with OA spend $61 \%$ of all waking hours in SB [32]. Finding strategies to address this knowledge-to-action gap are thus greatly needed since increasing MVPA and reducing SB among adults with OA may provide benefits for both physical and cognitive health.

One promising strategy for increasing MVPA and/ or reducing $\mathrm{SB}$ is consumer-available, wearable activity-monitoring technology. These devices present several distinct advantages as a MVPA promotion and SB reduction tool including: 1) adults typically perceive activity-monitors as useful [33]; 2) these devices incorporate multiple behavioural change strategies [34]; and 3) clinicians can readily use these devices to help promote behaviour change among their underactive patients [35]. Importantly, we recently determined that a wearable technology enabled counselling program for adults with knee OA increased MVPA by $25 \mathrm{~min} /$ day and improved OA symptoms [36]. Within this study, we included secondary measures to determine if increasing MVPA and/or reducing SB among adults with knee OA could also benefit cognitive function. Thus, the aim of the present paper is to determine whether this intervention to increase MVPA and reduce $\mathrm{SB}$ among adults with $\mathrm{OA}$ also improved cognitive function.

\section{Methods \\ Study design}

This study was a secondary analysis of Monitor-OA, a six month randomized controlled trial (RCT) examining the efficacy of a technology-enabled counselling intervention for increasing MVPA and reducing SB in people with knee OA [36]. The study occurred between November 1st 2015 and June 1st 2017. We used a randomized delayed-control design. In this 
study design, randomization determined the timing of when the intervention was provided (i.e., immediately vs. a 2-month delay).

\section{Participants}

We recruited community-dwelling adults from Vancouver, British Columbia who had a physician confirmed diagnosis of knee OA, or passed two criteria for early OA: 1) aged 50+ years; and 2) experienced knee pain during the previous year lasting $>28$ separate or consecutive days [37]. Participants were excluded if they 1) had been diagnosed with inflammatory arthritis, connective tissue diseases, fibromyalgia, or gout; 2) used anti-rheumatic drugs or gout medications; 3) had previously underwent knee arthroplasty, or were on the waitlist to receive total knee arthroplasty; 4) had suffered an acute knee injury in the past six months; 5) had a body mass index (BMI) of $>40 \mathrm{~kg} / \mathrm{m}^{2}$; 6) had received a steroid injection or a hyaluronate injection in the last 6 months; 7) were using medications which impaired physical activity tolerance (e.g., beta blockers), or had an inappropriate level of risk for increasing their physical activity. Participants were also excluded if they did not have access to a computer in their home, or did not have a personal email address. Potential participants completed the Physical Activity Readiness Questionnaire (PAR-Q) [38]. If a potential risk was identified by the PAR-Q, physician confirmation was required to ensure the participant was able to be physically active without supervision of a health professional.

The CONSORT (Consolidated Standards of Reporting Trials) flowchart in Fig. 1 shows the number of participants in the treatment arms at each stage of the study [39]. The research protocol was approved by the University of British Columbia Behavioural Research Ethics Board (Application number: H14-01762), and was published in ClinicalTrials.gov (NCT02315664).

\section{Measures}

Trained staff members administered all testing procedures. We assessed participants at baseline, 2 months, 4 months, and 6 months follow-up. In this paper we report data from baseline, 2 months, and 4 months.

\section{Demographics}

At baseline, we obtained general health history and demographics information by questionnaire. Height and weight

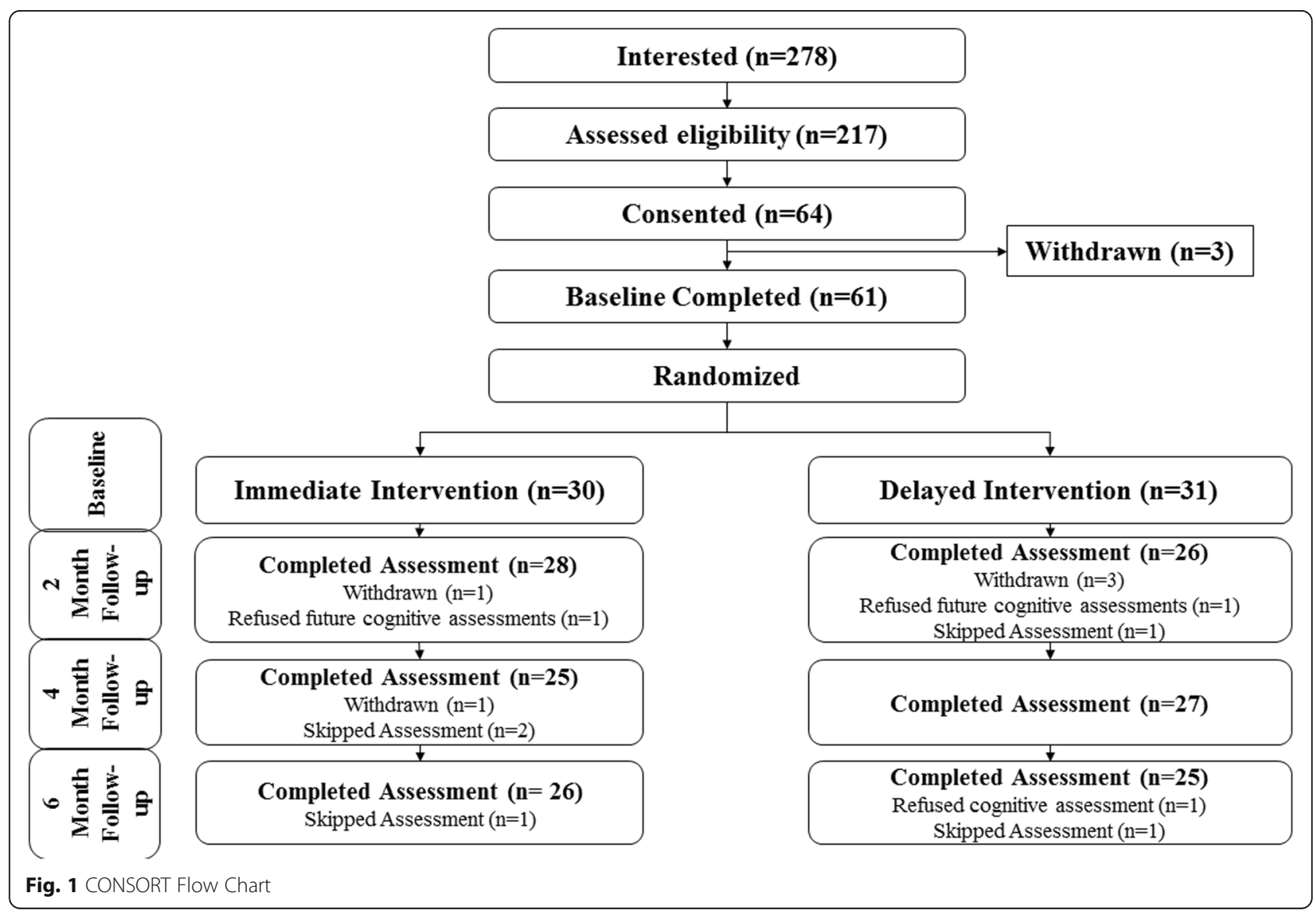


were ascertained using a calibrated stadiometer and an electronic scale, respectively. This information was used to determine BMI. In addition, we assessed global cognitive function at baseline using the Mini-Mental State Exam and the Montreal Cognitive Assessment [40, 41].

\section{MVPA and SB}

We measured MVPA and SB using the SenseWear Mini (Body Media, Pittsburgh, PA, USA), a multi-sensor monitor worn on the upper arm over the triceps [42]. Briefly, the device integrates tri-axial accelerometer data, physiological sensor data and personal demographic information to provide valid and reliable estimates of MVPA and SB [42-44]. Participants wore the device on the non-dominant arm for 7 days at each assessment. For our analyses, we examined time spent in MVPA in periods of 10 or more minutes, and time spent in SB in periods of 20 or more minutes.

\section{Cognitive function}

We measured cognitive function using the National Institutes of Health Toolbox Cognition Battery (NIHTB-CB) [45]. Briefly, the NIHTB-CB provides a brief, convenient set of computerized and standardized measures of cognitive function. We examined two specific cognitive subdomains: 1) episodic memory using the picture sequence memory task [46]; and 2) working memory using the list-sorting task [47]. Empirical evidence suggests increasing MVPA or reducing SB can influence each of these domains of cognitive function $[26,48]$. Briefly, the picture sequence memory task assesses episodic memory by having participants remember a sequence of actions embedded within a story. Participants re-arrange several pictures on the computer to match the sequence of events in the story. The list-sorting task assesses working memory by asking participants to repeat the names of orally-and visually-presented stimuli in order of size, from smallest largest. The number of items per set increases from one trial to the next and is discontinued once 2 trials of the same length are failed. Three trials of increasing length are completed. We recorded age-corrected scores for each measure.

\section{Randomization}

After completing the baseline assessment, eligible participants were randomly assigned to the Immediate Intervention (I-INT) or the Delayed Intervention (i.e. control; D-INT) in a 1:1 allocation ratio. Randomization was performed using computer-generated random numbers in variable block sizes. The D-INT received the same intervention as the I-INT after a 2-month wait.

\section{Intervention}

Details of the intervention have been described previously [36]. Briefly, the intervention consisted of participants attending a 1.5-h session, where they received: 1 ) a standardized group education session about the benefits of increasing MVPA and reducing SB; 2) a Fitbit ${ }^{\circ}$ Flex $^{\mathrm{Tw}}$; and 3) individual activity counselling with a physiotherapist. The education session was delivered in groups of 2-3 participants. The individual activity counselling session followed the Brief Action Planning approach [49]. The physiotherapist guided participants to identify their MVPA goals (e.g., begin resistance training, start cycling, join a walking group, etc.), develop an action plan (i.e., where they plan to perform their MVPA goal, how often, and for how long), identify barriers and solutions, and then rate their confidence in executing the plan. For SB, the physiotherapists began by asking participants to estimate their sitting time in a normal day. Participants were then asked to identify ways to break up their sitting time into shorter bouts.

Following the education session, participants were provided with a Fitbit Flex ${ }^{\mathrm{mm}}$ which they were instructed to wear $24 \mathrm{~h}$ /day except during water-based activity (i.e., swimming or bathing) or when charging the device. The Fitbit data were wirelessly synchronized with Fitbit's online Dashboard which could be viewed only by the participants and the study physiotherapist. During the intervention period, the physiotherapist reviewed the individual's MVPA on the Dashboard and progressively modified the activity goals during 4 biweekly phone calls. During these phone calls, we also monitored participant adherence to SB goals using self-report. Specifically, participants were asked at each biweekly phone call whether they fully met, partially met, or did not meet their SB goal. These goals were then modified as needed.

\section{Statistical analyses}

We conducted all statistical analyses using $\mathrm{R}$ version 3.3.1 in the lsmeans 2.26-3, lmerTest 2.0-33, and mice 2.46.0 packages. Descriptive statistics were used to summarize participant demographics. In order to account for missing data at each follow-up time point, we performed multiple imputation in the mice 2.46.0 package using predicted mean matching (5 imputations; 20 iterations each), and visually checked for convergence. All statistical models used pooled estimates from all 5 imputed data sets. Plots and graphs were created using ggplot2 2.2.1. Our statistical code can be found in Additional file 1.

\section{Main analyses}

We evaluated between-group differences (I-INT vs. D-INT) on the outcomes of interest following the primary intervention (i.e., Baseline - 2 Months) using the intention-to-treat principle, as per our primary outcomes paper [36]. Two separate analyses of covariance (ANCOVA) models were conducted, wherein cognitive performance at 2 months was the dependent variable 
and treatment group was the independent variable of interest; both models controlled for baseline cognitive performance. We estimated group mean changes in cognitive function and corresponding 95\% confidence intervals pooled across the 5 imputed datasets, as well as estimated group mean difference (with $95 \% \mathrm{CI}$ ) and estimated Cohen's $d$ effect size.

\section{Secondary analyses}

We also examined whether changes in MVPA or SB during the intervention were associated with changes in cognitive function. We created change scores (i.e., I-INT = Baseline -2 Months; D-INT = Baseline -4 Months) for MVPA, SB, the list-sorting task, and the picture sequence memory task. We performed four separate multiple linear regression models wherein changes in cognitive performance (i.e., list-sorting memory or picture sequence memory) during the intervention were specified as the dependent variable, and changes in MVPA (or SB) was specified as the independent variable of interest. Each model included 1) baseline score for the cognitive performance variable of interest; 2) baseline MVPA (or SB); and 3) treatment group as covariates of no interest. We report unstandardized beta values and standard errors. Given our sample size, and a two-tailed $\alpha=0.05$, we had $80 \%$ power to detect a two-sided correlation with a medium effect size of $|\rho|=0.34$ [50].

\section{Results}

\section{Participant characteristics}

From 2015 to 2016, 278 people indicated an interest to participate, and 64 met the eligibility criteria (Fig. 1). Of those, we recruited 61 participants (I-INT: $n=30$; D-INT: $n=31$ ). As described in Table 1 , there were no group differences in age (I-INT: 61.73 [SD 9.40] years;
D-INT: 62.61 [8.54]), sex (I-INT: 73.33\% female; D-INT: 90.32\%), BMI (I-INT: 29.16 [5.46] kg/m²; D-INT: 29.24 [4.82), or time spent in MVPA (I-INT: 83.44 [60.80] minutes/day; D-INT: 86.19 [86.19] minutes/day) and SB (I-INT: 681.96 [111.51] minutes/day; D-INT: 703.05 [161.17] minutes/day) at baseline. Participants in I-INT had a lower picture sequence memory score (I-INT: 102.04 [17.22]; D-INT: 112.53 [14.67]; $p=0.02$ ), but there were no differences between groups for list-sorting score (I-INT: 102.05 [17.22]; D-INT: 102.42 [14.64]).

\section{Changes in cognitive function}

Group differences in cognitive performance-accounting for baseline cognitive performance-are illustrated in Fig. 2. Briefly, there were no statistically significant differences between groups following the intervention. As described in Table 2, we calculated a small improvement of the I-INT compared to D-INT for picture sequence memory (estimated mean difference: 1.27; 95\% CI [9.27, 11.81]; $d=0.10$ ), and a small improvement of the D-INT compared to the I-INT for list-sorting memory (estimated mean difference: $-1.64 ; 95 \%$ CI $[-8.72,5.44]$; $d=-0.19)$.

\section{Correlation between MVPA and SB changes with changes in cognitive function}

The relationship between changes in MVPA and changes in cognitive function are illustrated in Fig. 3. There were no statistically significant relationships between changes in MVPA and changes in cognitive function. Increases in MVPA were correlated with changes in list-sorting memory in the expected direction $(B=0.04 ; 95 \%$ CI [$0.07,0.14]$ ), however changes in picture sequence memory appeared to be negatively correlated with increases in MVPA (B $=-0.02$; $95 \%$ CI $[-0.15,0.12])$.

Table 1 Baseline Characteristics

\begin{tabular}{|c|c|c|c|}
\hline Participant Characteristic & Immediate Intervention $(N=30)$ & Delayed Intervention $(N=31)$ & p \\
\hline Age & $61.73(9.40)$ & $62.61(8.54)$ & 0.70 \\
\hline \%Female & $73.33 \%$ & $90.32 \%$ & 0.16 \\
\hline \multicolumn{4}{|l|}{ Education } \\
\hline High school or less & $16.67 \%$ & $19.35 \%$ & \multirow[t]{3}{*}{0.92} \\
\hline Some university & $33.33 \%$ & $29.03 \%$ & \\
\hline University degree or higher & $50.00 \%$ & $51.61 \%$ & \\
\hline Body Mass Index (kg/m) & $29.16(5.46)$ & $29.24(4.82)$ & 0.95 \\
\hline Moderate-to-Vigorous Physical Activity (min/day) & $83.44(60.80)$ & $86.19(86.19)$ & 0.89 \\
\hline Sedentary Behaviour (min/day) & $681.96(111.51)$ & $703.05(161.17)$ & 0.55 \\
\hline Mini-Mental State Exam & $28.03(2.62)$ & $28.62(1.35)$ & 0.28 \\
\hline Montreal Cognitive Assessment & $27.27(2.53)$ & $26.24(2.86)$ & 0.15 \\
\hline List-Sorting Task & 102.05 (13.03) & $102.42(14.64)$ & 0.92 \\
\hline Picture Sequence Memory Task & $102.04(17.22)$ & $112.53(14.67)$ & 0.02 \\
\hline
\end{tabular}






The relationship between changes in SB and changes in cognitive function are illustrated in Fig. 4. There were no statistically significant relationships between changes in SB and changes in either picture sequence memory $(B=-0.01 ; 95 \% C I[-0.09,0.07])$ or list-sorting memory $(B=0.00 ; 95 \%$ CI $[-0.09,0.10])$.

\section{Discussion}

Although we previously determined this intervention can increase MVPA and improve quality of life among adults with knee OA [36], there does not appear to be sufficient evidence that our intervention can also improve cognitive function within this population. However, our results suggest that future research on the role of MVPA and SB for maintaining cognitive health among adults with $\mathrm{OA}$ is warranted. Given that most adults with OA are inactive [32], and thus more at risk to future cognitive decline [26,27], we believe that such research would be valuable. We now discuss potential explanations for our findings.

While there is strong evidence that MVPA in the form of exercise training can improve cognitive function [51], the results of community-based MVPA interventions to promote cognitive health have been far less conclusive [52]. Importantly, the effects of MVPA on cognitive function seem to be largest for individuals who are underactive, while regular MVPA may be mainly neuroprotective for individuals who are meeting current guidelines [53]. Our participants were already highly active at baseline, and thus changes in MVPA may have had limited impact on cognitive function due to high basal levels. Moreover, the high activity level of our sample at baseline suggests that the results may not be generalizable to most adults with $\mathrm{OA}-$ who are sedentary. In order to first determine the efficacy of MVPA as a treatment for maintaining cognitive health for adults with OA, future trials should therefore recruit less active individuals since they 1) are more generalizable to the OA population; and 2) will more likely reap the most benefits from increasing their MVPA.

To date, most of the evidence examining how SB impacts cognitive health comes from epidemiological data $[26,29,30]$. Our study is the first to examine if an intervention to reduce $\mathrm{SB}$ can improve cognitive health among adults with OA. The results do not appear to suggest reductions in SB are associated with improvements in cognitive function; however, our intervention did not significantly reduce time spent in SB [36]. Preliminary evidence does suggest reductions in sedentary time may reverse the deleterious physiological effects of $\mathrm{SB}-$ such as impaired glucose and lipid metabolism [54]. Healthy glucose and lipid metabolism are strongly linked to better cognitive health [55]. Thus, while our results cannot determine

Table 2 Changes in cognitive function (Baseline - 2 Months) by treatment group

\begin{tabular}{lllll}
\hline Variable & $\begin{array}{l}\text { Immediate Intervention Group } \\
\text { Mean [95\% Cl] }\end{array}$ & $\begin{array}{l}\text { Delayed Intervention Group } \\
\text { Mean [95\% Cl] }\end{array}$ & Estimated Mean Group Difference [95\% Cl] & $d$ \\
\hline List Sorting Task & $2.90[-1.55,7.35]$ & $4.53[-0.53,9.59]$ & $-1.64[-8.72,5.44]$ & -0.19 \\
Picture Sequence Memory Task & $4.21[-2.55,10.97]$ & $2.95[-6.36,12.26]$ & $1.27[-9.27,11.81]$ & 0.10 \\
\hline
\end{tabular}

Note: All estimates adjusted for cognitive score at baseline 


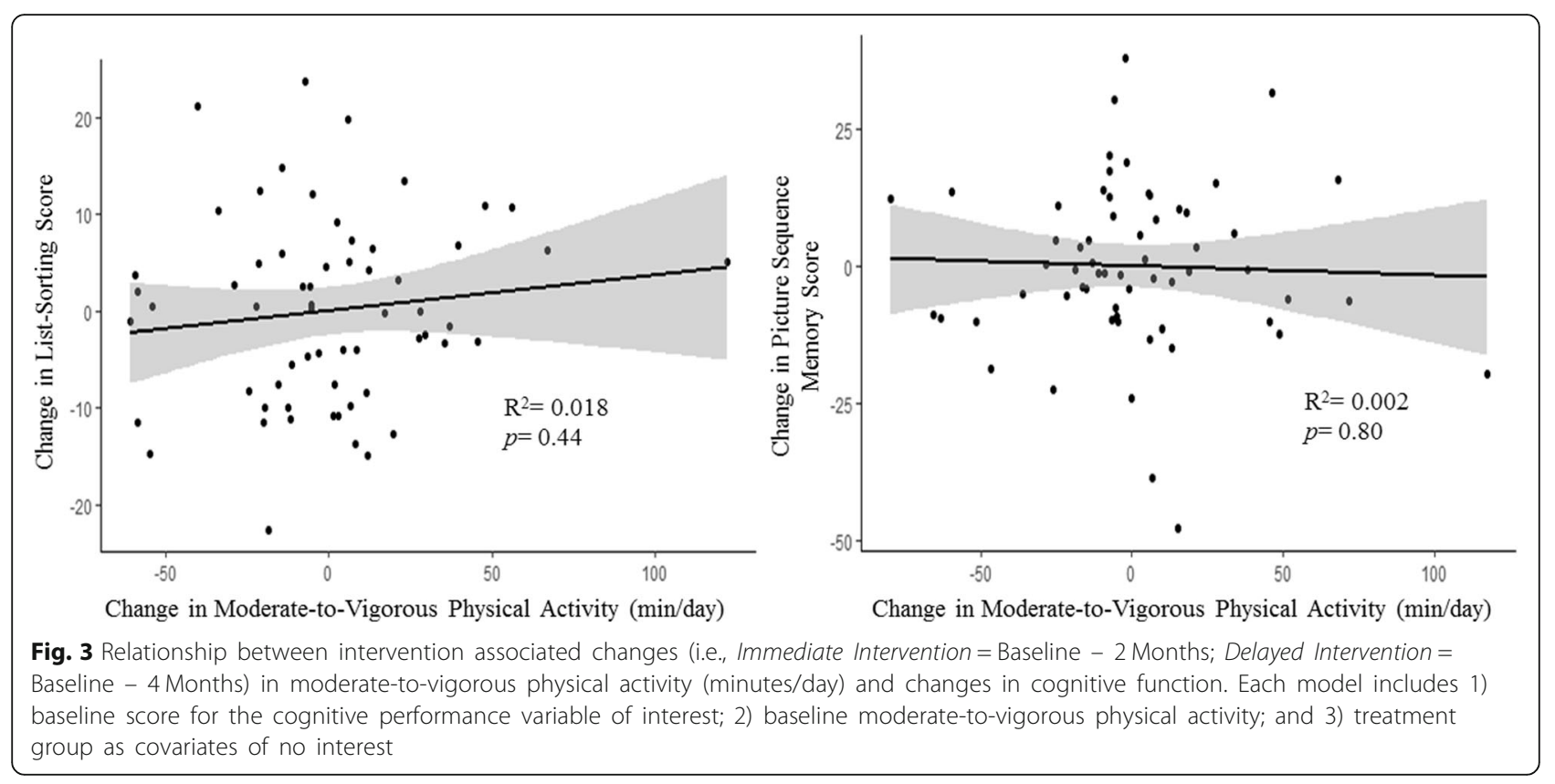

whether reducing SB can improve cognitive function, there does appear to be a plausible mechanism by which reduced SB may benefit cognitive function.

This was a secondary analysis of a proof-of-concept $\mathrm{RCT}$, and thus we think the logical next step is for an adequately powered RCT to determine the efficacy of this intervention to promote cognitive health among adults with OA. A recent meta-analysis suggests that MVPA in the form of exercise training has a modest effect size on cognitive function of $d=0.29$ [51]. Based on this effect size, we post-hoc investigated the necessary sample size to perform an adequately powered RCT to improve cognitive function using G*Power 3.1 [50]. In order to detect an effect size of the magnitude suggested by Northey and colleagues [51], we would need at least 376 participants to achieve $80 \%$ power (assuming a two-sided $\alpha=0.05$ ). The study we report herein was thus under-powered, however we would expect the effect sizes for this intervention to increase through two simple strategies. First, future studies should exclude adults that are already physically active since the largest effects of MVPA on health occur for individuals who are inactive [56]. Second, increasing the length of time between assessment points would help reduce the potential for practice effects to occur on cognitive tests [57], and provide adequate time for eliciting changes in

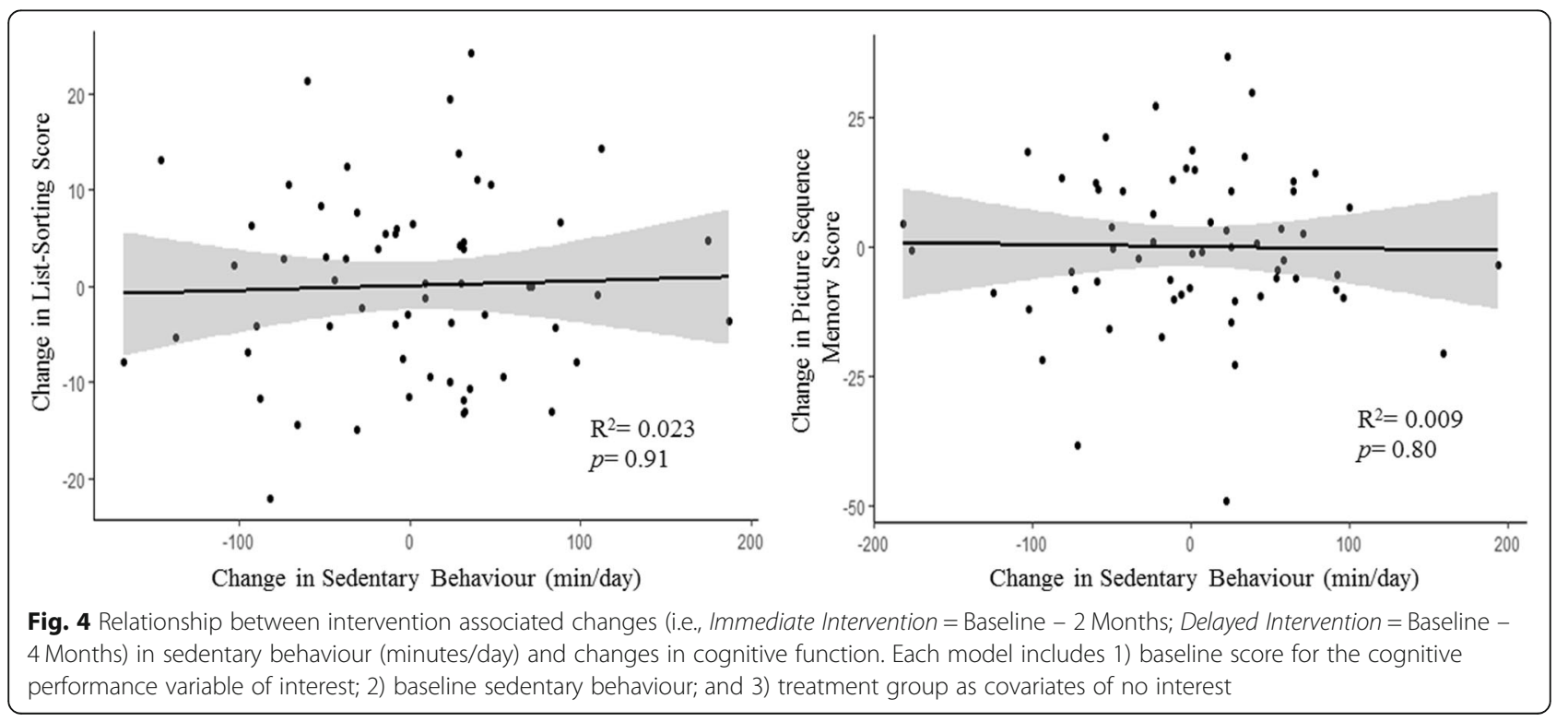


cognitive function which evidence suggests are larger after at least 6 months of increased MVPA [48].

\section{Clinical applications}

Although we did not find that our intervention significantly improved cognitive function, there are several potential clinical applications to our study. First, we previously demonstrated that clinicians (i.e., physical therapists) can use consumer-available wearable activity-monitors such as a Fitbit to promote MVPA and reduce SB among their patients with OA [36]. Given the health care system has an untapped capacity for promoting changes in MVPA and $\mathrm{SB}$, which to date has not been fully developed [58], a first step towards harnessing this potential to promote behaviour change is for clinicians to track their patients' MVPA and SB using wearable activity-monitors. At minimum, clinicians should query about activity during their consultations with OA patients [59].

Secondly, both OA and SB may increase the risk of cognitive impairment and dementia [7-9, 26, 29, 30]. In contrast, engagement in MVPA reduces dementia risk and promotes overall cognitive and physical health [14-16, 27, 28]. Adults with OA should therefore be encouraged to adhere to the current public health recommendations of engaging in $\geq 150 \mathrm{~min} /$ week of MVPA and limiting their SB as much as possible [26]. Importantly, $87 \%$ of adults with OA are underactive [31] and adults with OA spend an average of $61 \%$ of the day in SB [32]. MVPA promotion and $\mathrm{SB}$ reduction may thus be particularly important for physical and cognitive health in adults with OA.

\section{Limitations and future research}

This was a secondary outcomes analysis, and thus our findings are a preliminary investigation of whether increasing MVPA and reducing SB can improve cognitive function in adults with OA. While the SenseWear Mini provides valid and reliable estimates of energy expenditure for both younger and older adults [42, 60], which can be used to derive time spent in MVPA and time spent in SB, we cannot determine whether time estimated as SB was actually spent sitting or lying down. Hence, future studies to examine changes in SB should use measures of body posture such as the activPAL [61], which can accurately determine whether a person is sitting, standing, or walking.

We did not collect information on medication use, however our participants were community-dwelling adults who were healthy enough to start a physical activity program at study entry. We also did not exclude participants based on current activity level and hence the results may not be generalizable to most people with $\mathrm{OA}$-who are often sedentary. Given the high activity level of our participants, the effects of increased MVPA and reduced SB on cognitive function may have been attenuated. We therefore suggest future studies should recruit underactive adults with OA, since these individuals are likely to benefit from increased MVPA and reduced SB.

There is growing evidence that the effects of MVPA (and potentially $\mathrm{SB}$ ) are moderated by age and sex [62, 63], however due to our small sample size, it was not feasible for us to control for age and sex within our analyses. As detailed previously [36], our intervention did not reduce SB which is perhaps due to several shortcomings of the counselling program, which we have rectified. Specifically, the intervention now includes a new SB counselling strategy, and a Fitbit-compatible web app with enhances functionality for setting goals and rewarding behaviours that break up prolonged sitting [64]. This paradigm is currently being tested in a RCT (ClinicalTrial.gov identifier: NCT02554474) involving people with rheumatoid arthritis and systematic lupus erythematosus [65].

\section{Conclusion}

While strong evidence indicates that increasing MVPA and reducing $\mathrm{SB}$ can positively impact OA symptoms, it is not yet clear whether increasing MVPA and reducing SB can also promote cognitive health among this population. However, increasing MVPA and reducing SB among adults with OA should be a public health priority since it can help maintain physical health and reduce the risk of cognitive impairment and dementia. Clinicians should therefore take the time to counsel their patients with OA to engage in $\geq 150 \mathrm{~min} /$ week of MVPA and limit their SB in order to promote physical and cognitive health.

\section{Additional file}

Additional file 1: $\mathrm{R}$ Code. (DOCX $15 \mathrm{~kb})$

\section{Abbreviations}

BMI: Body Mass Index; D-INT: Delayed Intervention; I-INT: Immediate Intervention; METS: Metabolic Equivalents; MVPA: Moderate-to-Vigorous Physical Activity; NIHTB-CB: National Institutes of Health Toolbox Cognition Battery; OA: Osteoarthritis; PACAP: Pituitary Adenylate Cyclase-Activating Peptide; PAR-Q: Physical Activity Readiness Questionnaire; RCT: Randomized Controlled Trial; SB: Sedentary Behaviour

\section{Acknowledgements}

We would like to thank the Track-OA research staff for their assistance with the intervention and data collection.

\section{Author contributions}

RSF wrote the first draft of the manuscript. LL and TLA conceived the study concept and design. RSF and JRB performed the data analysis and interpreted the results. JRB, LL, PCYC, LMF and TLA wrote portions of the manuscript and provided critical review. All authors have read and approve of the final manuscript.

\section{Funding}

Funding for this project was provided by a Canadian Institutes of Health Research Operational Grant (F14-03974), and by the Jack Brown \& Family Alzheimer's Research Foundation. The funding bodies did not play a role in 
the study design. RSF is funded by the University of British Columbia Rehabilitation Sciences Scholarship, the Omer H. Patrick II Memorial Prize, and the Louise McGregor Memorial Award in Neurorehabilitation. TLA is a Canada Research Chair in Physical Activity, Mobility, and Cognitive Heatlth. LCL is a Canada Research Chair in Patient-Oriented Knowledge Translation and holds the Harold Robinson / Arthritis Society Chair in Arthritic Diseases.

\section{Availability of data and materials}

The datasets during and/or analysed during the current study available from the corresponding author on reasonable request.

\section{Ethics approval and consent to participate}

This study was was approved by Vancouver Coastal Health Research Institute and the University of British Columbia's Clinical Research Ethics Board (H1401762). All participants provided written informed consent.

\section{Consent for publication}

Not applicable.

\section{Competing interests}

The authors declare that they have no competing interests.

\section{Publisher's Note}

Springer Nature remains neutral with regard to jurisdictional claims in published maps and institutional affiliations.

\section{Author details}

${ }^{1}$ Faculty of Medicine, Aging, Mobility and Cognitive Neuroscience Laboratory, Djavad Mowafaghian Centre for Brain Health, Department of Physical Therapy, University of British Columbia, 212-2177 Wesbrook Mall, Vancouver, BC V6T 1Z3, Canada. 'Faculty of Medicine, Arthritis Research Canada, University of British Columbia, Vancouver, Canada.

\section{Received: 7 September 2018 Accepted: 3 December 2018} Published online: 21 December 2018

\section{References}

1. Bombardier C, Hawker G, Mosher D. The impact of arthritis in Canada: today and over the next 30 years. Toronto: Arthritis Alliance of Canada. 2011;2011. http://www.arthritisalliance.ca/images/PDF/eng/Initiatives/20111022_2200_ impact_of_arthritis.pdf.

2. Laupattarakasem W, Laopaiboon M, Laupattarakasem P, Sumananont C. Arthroscopic debridement for knee osteoarthritis. Cochrane Database Syst Rev. 2008;1:Cd005118. https://doi.org/10.1002/14651858.CD005118.pub2.

3. Husted JA, Tom BD, Farewell VT, Schentag CT, Gladman DDA. Longitudinal study of the effect of disease activity and clinical damage on physical function over the course of psoriatic arthritis: does the effect change over time? Arthritis Rheum. 2007:56(3):840-9.

4. Sturnieks DL, Tiedemann A, Chapman K, Munro B, Murray SM, Lord SR. Physiological risk factors for falls in older Peoplen with lower limb arthritis. J Rheumatol. 2004;31(11):2272-9.

5. Hatfield GL, Hubley-Kozey CL, Wilson JLA, Dunbar MJ. The effect of total knee arthroplasty on knee joint kinematics and kinetics during gait. J Arthroplast. 2011;26(2):309-18.

6. Beswick AD, Wylde V, Gooberman-Hill R, Blom A, Dieppe P. What proportion of patients report long-term pain after total hip or knee replacement for osteoarthritis? A systematic review of prospective studies in unselected patients. BMJ Open. 2012;2(1):e000435.

7. Huang S-W, Wang W-T, Chou L-C, Liao C-D, Liou T-H, Lin H-W. Osteoarthritis increases the risk of dementia: a nationwide cohort study in Taiwan. Sci Rep. 2015;5:10145.

8. Baker NA, Barbour KE, Helmick CG, Zack M, Al Snih S. Arthritis and cognitive impairment in older adults. Rheumatol Int. 2017;37(6):955-61.

9. Kyrkanides S, Tallents RH, Jen-nie HM, Olschowka ME, Johnson R, Yang M, et al. Osteoarthritis accelerates and exacerbates Alzheimer's disease pathology in mice. J Neuroinflammation. 2011;8(1):112.

10. Prevalence of doctor-diagnosed arthritis and arthritis-attributable activity limitation--United States. 2010-2012. Morb Mortal Wkly Rep. 2013;62(44): 869-73.

11. World Health Organization, Alzheimer's Disease International. Dementia: A Public Health Authority. 2012.
12. Lee J, Chang RW, Ehrlich-Jones L, Kwoh CK, Nevitt M, Semanik PA, et al. Sedentary behavior and physical function: objective evidence from the osteoarthritis initiative. Arthritis Care \& Research. 2015;67(3):366-73.

13. Song J, Lindquist LA, Chang RW, Semanik PA, Ehrlich-Jones LS, Lee J, et al. Sedentary behavior as a risk factor for physical frailty independent of moderate activity: results from the osteoarthritis initiative. Am J Public Health. 2015;105(7):1439-45.

14. Brosseau L, MacLeay L, Robinson V, Wells G, Tugwell P. Intensity of exercise for the treatment of osteoarthritis. Cochrane Database Syst Rev. 2003;2.

15. Zhang W, Moskowitz R, Nuki G, Abramson S, Altman R, Arden N, et al. OARSI recommendations for the management of hip and knee osteoarthritis, part I: critical appraisal of existing treatment guidelines and systematic review of current research evidence. Osteoarthr Cartil. 2007;15(9): 981-1000.

16. Brosseau L, Wells GA, Tugwell P, Egan M, Dubouloz C, Casimiro L, et al. Ottawa panel evidence-based clinical practice guidelines for therapeutic exercises and manual therapy in the management of osteoarthritis. Phys Ther. 2005;85(9):907-71.

17. Giunta S, Castorina A, Marzagalli R, Szychlinska MA, Pichler K, Mobasheri A, Musumeci G. Ameliorative effects of PACAP against cartilage degeneration. Morphological, immunohistochemical and biochemical evidence from in vivo and in vitro models of rat osteoarthritis. Int J Mol Sci. 2015;16(3):5922-44.

18. Szychlinska MA, Leonardi R, Al-Qahtani M, Mobasheri A, Musumeci G. Altered joint tribology in osteoarthritis: reduced lubricin synthesis due to the inflammatory process. New horizons for therapeutic approaches Annals of Physical and Rehabilitation Medicine. 2016;59(3):149-56.

19. Musumeci G, Loreto C, Leonardi R, Castorina S, Giunta S, Carnazza ML, et al. The effects of physical activity on apoptosis and lubricin expression in articular cartilage in rats with glucocorticoid-induced osteoporosis. J Bone Miner Metab. 2013;31(3):274-84.

20. Musumeci G, Castrogiovanni P, Trovato FM, Imbesi R, Giunta S, Szychlinska $M A$, et al. Physical activity ameliorates cartilage degeneration in a rat model of aging: a study on lubricin expression. Scand J Med Sci Sports. 2015;25(2): e222-30.

21. Musumeci G, Trovato FM, Pichler K, Weinberg AM, Loreto C, Castrogiovanni P. Extra-virgin olive oil diet and mild physical activity prevent cartilage degeneration in an osteoarthritis model: an in vivo and in vitro study on lubricin expression. J Nutr Biochem. 2013;24(12):2064-75.

22. Juhász T, Szentléleky E, Somogyi CS, Takács R, Dobrosi N, Engler M, et al. Pituitary adenylate cyclase activating polypeptide (PACAP) pathway is induced by mechanical load and reduces the activity of hedgehog signaling in chondrogenic micromass cell cultures. Int J Mol Sci. 2015;16(8): 17344-67.

23. Suomi R, Collier D. Effects of arthritis exercise programs on functional fitness and perceived activities of daily living measures in older adults with arthritis. Archives of Physical Medicine Rehabilitation. 2003:84(11):1589-94.

24. Barnett A, Smith B, Lord SR, Williams M, Baumand A. Community-based group exercise improves balance and reduces falls in at-risk older people: a randomised controlled trial. Age Ageing. 2003;32(4):407-14.

25. Semble EL, Loeser RF, Wise CM. Therapeutic exercise for rheumatoid arthritis and osteoarthritis. Semin Arthritis Rheum. 1990;20(1):32-40.

26. Falck RS, Davis JC, Liu-Ambrose T. What is the association between sedentary behaviour and cognitive function? A systematic review British Journal of Sports Medicine. 2017:51:800-11.

27. Hamer M, Chida Y. Physical activity and risk of neurodegenerative disease: a systematic review of prospective evidence. Psychol Med. 2009;39(1):3-11.

28. Adlard PA, Perreau VM, Pop V, Cotman CW. Voluntary exercise decreases amyloid load in a transgenic model of Alzheimer's disease. J Neurosci. 2005; 25(17):4217-21.

29. Vaynman S, Gomez-Pinilla F. Revenge of the "sit": how lifestyle impacts neuronal and cognitive health through molecular systems that interface energy metabolism with neuronal plasticity. J of Neurosci Res. 2006;84(4): 699-715.

30. Voss MW, Carr LJ, Clark R, Weng T. Revenge of the "sit" II: does lifestyle impact neuronal and cognitive health through distinct mechanisms associated with sedentary behavior and physical activity? Ment Health and Phys Act. 2014;7(1):9-24.

31. Wallis JA, Webster KE, Levinger P, Taylor NF. What proportion of people with hip and knee osteoarthritis meet physical activity guidelines? A systematic review and meta-analysis. Osteoarthr Cartil. 2013;21 (11):1648-59. 
32. Sliepen M, Mauricio E, Lipperts M, Grimm B, Rosenbaum D. Objective assessment of physical activity and sedentary behaviour in knee osteoarthritis patients-beyond daily steps and total sedentary time. BMC Musculoskelet Disord. 2018:19(1):64.

33. Mercer K, Giangregorio L, Schneider E, Chilana P, Li M, Grindrod K. Acceptance of commercially available wearable activity trackers among adults aged over 50 and with chronic illness: a mixed-methods evaluation. J Med Internet Res. 2016:4(1)

34. Mercer K, Li M, Giangregorio L, Burns C, Grindrod K. Behavior change techniques present in wearable activity trackers: a critical analysis. J Med Internet Res. 2016:4(2).

35. Falck RS, Landry GJ, Best JR, Davis JC, Chiu BK, Liu-Ambrose T. crosssectional relationships of physical activity and sedentary behavior with cognitive function in older adults with probable mild cognitive impairment. Phys Ther. 2017

36. Li LC, Sayre EC, Xie H, Falck RS, Best JR, Liu-Ambrose T, et al. efficacy of a community-based technology-enabled physical activity counselling program for people with knee osteoarthritis: a proof-of-concept study. J Med Internet Res. 2018;20(4):e159.

37. Marra CA, Cibere J, Tsuyuki RT, Soon JA, Esdaile JM, Gastonguay L, et al. improving osteoarthritis detection in the community: pharmacist identification of new, diagnostically confirmed osteoarthritis. Arthritis Care \& Research. 2007:57(7):1238-44.

38. Cardinal BJ, Cardinal MK. Preparticipation physical activity screening within a racially diverse, older adult sample: comparison of the original and revised physical activity readiness questionnaires. Res Q Exerc Sport. 2000;71(3):302-7.

39. Begg C, Cho M, Eastwood S, Horton R, Moher D, Olkin I, et al. Improving the quality of reporting of randomized controlled trials: the CONSORT statement. J Am Med Assoc. 1996;276(8):637-9

40. Folstein MF, Folstein SE, McHugh PR. "Mini-mental state". A practical method for grading the cognitive state of patients for the clinician. J Psychiatr Res. 1975;12(3):189-98.

41. Nasreddine ZS, Phillips NA, Bédirian V, Charbonneau S, Whitehead V, Collin I, et al. The Montreal cognitive assessment, MoCA: a brief screening tool for mild cognitive impairment. Journal of the American Geriatric Society. 2005; 53(4):695-9.

42. Johannsen DL, Calabro MA, Stewart J, Franke W, Rood JC, Welk GJ. Accuracy of armband monitors for measuring daily energy expenditure in health adults. Medicine \& Science in Sport \& Exercise. 2011;42(11):2134-40.

43. Reece JD, Barry V, Fuller DK, Caputo J. Validation of the SenseWear armband as a measure of sedentary behavior and light activity. J Phys Act Health. 2015;12(9):1229-37.

44. Tierney M, Fraser A, Purtill H, Kennedy N. Study to determine the criterion validity of the SenseWear armband as a measure of physical activity in people with rheumatoid arthritis. Arthritis Care \& Research. 2013;65(6):888-95.

45. Weintraub S. Cognition assessment using the NIH toolbox. Neurology. 2013; 80(S3):S54-64

46. Dikmen SS, Bauer PJ, Weintraub S, Mungas D, Slotkin J, Beaumont JL, et al. Measuring episodic memory across the lifespan: $\mathrm{NIH}$ toolbox picture sequence memory test. J Int Neuropsychol Soc. 2014;20(6):611-9.

47. Tulsky DS, Carlozzi N, Chiaravalloti ND, Beaumont JL, Kisala PA, Mungas D, et al. NIH toolbox cognition battery (NIHTB-CB): list sorting test to measure working memory. J Int Neuropsychol Soc. 2014;20(6):599-610.

48. Colcombe $\mathrm{S}$, Kramer AF. Fitness effects on the cognitive function of older adults: a meta-analytic study. Psychol Sci. 2003;14(2):125-30.

49. Gutnick D, Reims K, Davis C, Gainforth H, Jay M, Cole S. Brief action planning to facilitate behavior change and support patient selfmanagement. J Clin Outcomes Manag. 2014;21(1):18-29.

50. Faul F, Erdfelder E, Lang A-G, Buchner A. G* power 3: a flexible statistical power analysis program for the social, behavioral, and biomedical sciences. Behavioral Research Methods. 2007;39(2):175-91.

51. Northey JM, Cherbuin N, Pumpa KL, Smee DJ, Rattray B. Exercise interventions for cognitive function in adults older than 50: a systematic review with meta-analysis. Br J Sports Med. 2018;52:154-60.

52. Sink KM, Espeland MA, Castro CM, Church T, Cohen R, Dodson JA, et al. Effect of a 24-month physical activity intervention vs health education on cognitive outcomes in sedentary older adults: the LIFE randomized trial. J Am Med Assoc. 2015;314(8):781-90.

53. Bherer L, Erickson Kl, Liu-Ambrose T. a review of the effects of physical activity and exercise on cognitive and brain functions in older adults. Journal of Aging Research. 2013;2013:657508.
54. Tremblay MS, Colley RC, Saunders TJ, Healy GN, Owen N. Physiological and health implications of a sedentary lifestyle. Appl Physiol Nutr Metab. 2010; 35(6):725-40.

55. Craft S. The role of metabolic disorders in Alzheimer disease and vascular dementia: two roads converged. Arch Neurol. 2009;66(3):300-5.

56. Physical Activity Guidelines Advisory Committee (2008). Physical activity guidelines advisory committee report, 2008. Washington: DC: US Department of Health and Human Services; 2008. p. A1-H14.

57. Falleti MG, Maruff P, Collie A, Darby DG. Practice effects associated with the repeated assessment of cognitive function using the CogState battery at 10-minute, one week and one month test-retest intervals. J Clin Exp Neuropsychol. 2006;28(7):1095-112.

58. Berra K, Rippe J, Manson JE. Making physical activity counseling a priority in clinical practice: the time for action is now. J Am Med Assoc. 2015;314(24):2617-8.

59. Sallis R. Developing healthcare systems to support exercise: exercise as the fifth vital sign. Br J Sports Med. 2011:45(6):473-4.

60. Mackey DC, Manini TM, Schoeller DA, Koster A, Glynn NW, Goodpaster BH, Satterfield S, Newman AB, Harris TB, Cummings SR, Health, Aging, and body composition study. Validation of an armband to measure daily energy expenditure in older adults. Journals of gerontology series a: biomedical sciences and medical Sciences. 2011:66(10):1108-13.

61. Koster A, Shiroma EJ, Caserotti P, Matthews CE, Chen KY, Glynn NW, Harris TB. Comparison of sedentary estimates between activPAL and hip-and wrist-worn ActiGraph. Med Sci Sports Exerc. 2016;48(8):1514.

62. Physical Activity Guidelines Advisory Committee. Physical activity guidelines advisory committee scientific report. Washington, DC: US Department of Health and Human Services; 2018.

63. Barha CK, Davis JC, Falck RS, Nagamatsu LS, Liu-Ambrose T. Sex differences in exercise efficacy to improve cognition: a systematic review and metaanalysis of randomized controlled trials in older humans. Front Neuroendocrinol. 2017:46:71-85.

64. Gupta A, Tong X, Shaw C, Li L, Feehan L, editors. FitViz: A Personal Informatics Tool for Self-management of Rheumatoid Arthritis. International Conference on Human-Computer Interaction; 2017: Springer.

65. Li LC, Feehan LM, Shaw C, Xie H, Sayre EC, Aviña-Zubeita A, et al. A technology-enabled counselling program versus a delayed treatment control to support physical activity participation in people with inflammatory arthritis: study protocol for the OPAM-IA randomized controlled trial. BMC Rheumatology. 2017;1(1):6.
Ready to submit your research? Choose BMC and benefit from:
- fast, convenient online submission
- thorough peer review by experienced researchers in your field
- rapid publication on acceptance
- support for research data, including large and complex data types
- gold Open Access which fosters wider collaboration and increased citations
- maximum visibility for your research: over $100 \mathrm{M}$ website views per year
At BMC, research is always in progress.
Learn more biomedcentral.com/submissions 